\title{
Cerebrospinal fluid procalcitonin predicts Gram-negative bacterial meningitis in patients with empiric antibiotic pretreatment
}

\author{
Wen $\mathrm{Li}^{1 \dagger}$, Fang Yuan ${ }^{1 \dagger}$, Xiaolong Sun ${ }^{2}$, Zhihan Zhao ${ }^{1}$, Yaoyao Zhang ${ }^{1}$ and Wen Jiang ${ }^{1 *}$
}

Keywords: Bacterial meningitis, Gram-negative, Procalcitonin, Diagnostic biomarker

\section{Dear Editor,}

The identification of pathogenic bacteria facilitates better targeted treatments for bacterial meningitis (BM). However, early differentiation of Gram-positive and Gram-negative BM is a persisting challenge, especially in patients with empiric antibiotic pretreatment. Serum procalcitonin (PCT) was reported to be associated with Gram-negative bacterium in bloodstream infections [1]. Our previous study demonstrated that CSF-PCT was a superior diagnostic biomarker for $\mathrm{BM}$ even in patients who had received empiric antibiotic pretreatment [2]. In this study, we aimed to evaluate the discriminative power of CSF-PCT for Gram-negative BM.

Consecutive patients with BM [3] admitted to neurological intensive care unit (N-ICU) at Xijing Hospital between October 2013 and April 2018 were included in this study. A total of 225 patients with suspected meningitis/encephalitis were screened. One hundred fourteen patients with tuberculous meningitis or viral meningitis/encephalitis or autoimmune encephalitis and 21 patients with unknown etiology were excluded. Ninety patients were diagnosed with $\mathrm{BM}$, among them, 59 had definite pathogenic bacteria identified by CSF culture and Gram stain: 33 (56\%) patients were Gram-positive, and 26 (44\%) patients were Gram-negative (Additional file 1: Figure S1). Of these 59 patients, $25(42 \%)$ cases were community-acquired and $34(58 \%)$ cases were nosocomial BM (Additional file 1: Table S1). Bacterial etiologies were presented in Additional file 1: Table S2. All the participants had empiric antibiotic pretreatments before admission to our N-ICU. Demographics, severity, clinical characteristics, treatments, and the time from CSF-PCT test to onset of BM were balanced in Gram-positive group and Gram-negative group (Additional file 1: Table S3). The CSF-PCT level of Gram-negative patients was significantly higher than that of Gram-positive patients $(0.5[0.2-1.6]$ versus 0.1 [0.1-0.3] $\mathrm{ng} / \mathrm{ml}, p<0.001$ ) (Table 1, Fig. 1). There were no significant differences of other biomarkers between Gram-positive and Gram-negative group (Additional file 1: Figure S2). CSF-PCT yielded an area under the curve (AUC) of 0.85 (95\% CI, 0.73 to 0.93), which was superior to serum PCT, CSF/serum C-reactive protein (CRP) (Fig. 1).

The early identification for the pathogenic bacteria of $\mathrm{BM}$ is of great importance to the rational use of antibiotics. However, meningitis with a negative Gram stain poses a diagnostic and therapeutic dilemma, especially in developing countries like China [4]. CSF and serum CRP were previously indicated to be associated with Gram-negative BM [5], but in patients

* Correspondence: jiangwen@fmmu.edu.cn

tWen Li and Fang Yuan contributed equally to this work.

${ }^{1}$ Department of Neurology, Xijing Hospital, Fourth Military Medical University, Xi'an 710032, People's Republic of China

Full list of author information is available at the end of the article 

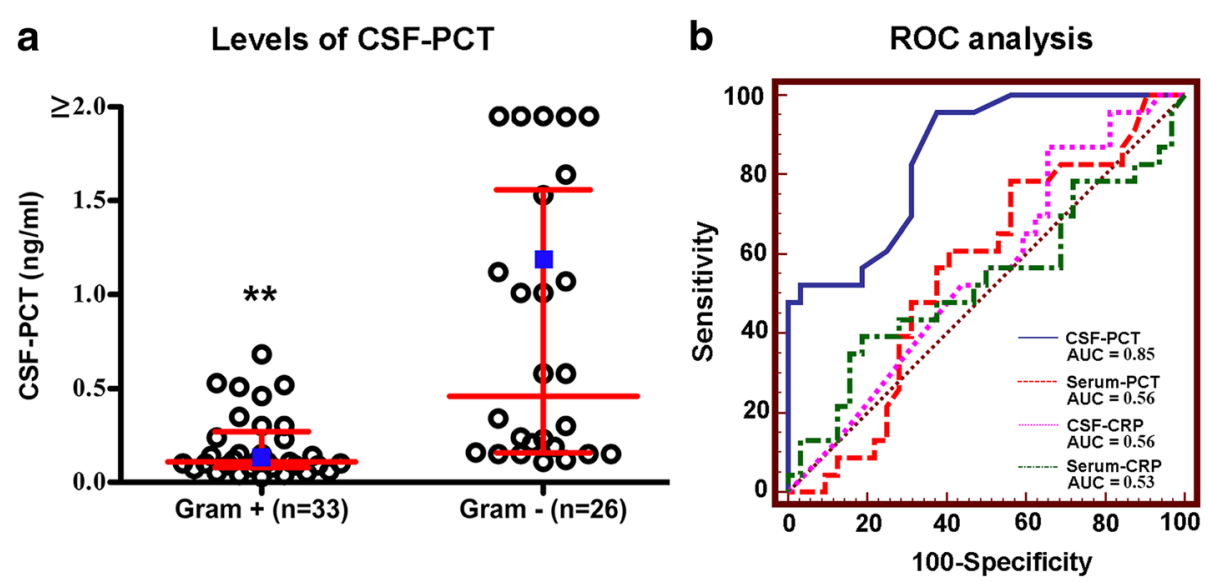

Fig. 1 Diagnostic value of CSF-PCT for Gram-negative BM. a Levels of CSF-PCT in Gram-negative and Gram-positive BM. The red lines and blue squares correspond to the group interquartile ranges and averages, respectively. ${ }^{* *} P<0.001$. b ROC curves of CSF/serum PCT, CSF/serum CRP for predicting Gram-negative BM. CSF-PCT: AUC = 0.85 ( $95 \%$ Cl, 0.73 to 0.93); serum PCT: AUC = 0.56 (95\% Cl, 0.42 to 0.69); CSF-CRP: AUC = 0.56 (95\% Cl, 0.42 to 0.70); serum CRP: AUC $=0.53$ (95\% Cl, 0.39 to 0.67). Comparisons of AUC: CSF-PCT vs serum PCT, $P<0.0001$; CSF-PCT vs CSF-CRP, $P=0.0034 ;$ CSF-PCT vs serum CRP, $P=0.0005$

Table 1 CSF and serum data

\begin{tabular}{|c|c|c|c|c|}
\hline & Total $(n=59)$ & Gram-positive $(n=33)$ & Gram-negative $(n=26)$ & $P$ value \\
\hline \multicolumn{5}{|l|}{ CSF } \\
\hline $\mathrm{PCT}, \mathrm{ng} / \mathrm{mL}$ & $0.2(0.1-0.5)$ & $0.1(0.1-0.3)$ & $0.5(0.2-1.6)$ & $<0.001$ \\
\hline Leukocyte, cells $/ \mathrm{mm}^{3}$ & $230.0(99.0-1790.0)$ & $214.0(105.5-1970.0)$ & 237.5 (56.0-1870.0) & 0.54 \\
\hline Neutrophils, cells $/ \mathrm{mm}^{3}$ & $154.1(13.2-1432.0)$ & $147.6(7.0-1726.5)$ & $165.0(23.0-1768.9)$ & 0.91 \\
\hline Lymphocyte, cells/mm³ & $50.4(20.1-114.7)$ & $52.1(17.4-150.6)$ & $44.3(23.3-100.5)$ & 0.71 \\
\hline Protein, g/L & $1.6(0.7-2.9)$ & $1.6(0.8-2.9)$ & $1.6(0.7-3.0)$ & 0.95 \\
\hline Glucose, mg/dL & $53.3(37.3-72.0)$ & $54.0(38.9-74.7)$ & $48.9(34.7-69.4)$ & 0.29 \\
\hline$C R P, m g / L$ & $0.3(0.2-0.9)$ & $0.3(0.2-1.0)$ & $0.3(0.2-0.8)$ & 0.82 \\
\hline First pressure, $\mathrm{cmH}_{2} \mathrm{O}$ & 157.5 (130.0-230.0) & $150.0(127.5-205.0)$ & $180.0(142.5-285.0)$ & 0.12 \\
\hline Last pressure, $\mathrm{CmH}_{2} \mathrm{O}$ & $95.0(70.0-130.0)$ & $90.0(70.0-128.8)$ & $100.0(72.5-167.5)$ & 0.26 \\
\hline \multicolumn{5}{|l|}{ Serum } \\
\hline $\mathrm{PCT}, \mathrm{ng} / \mathrm{mL}$ & $0.6(0.1-3.1)$ & $0.4(0.1-4.3)$ & $0.8(0.1-2.7)$ & 0.61 \\
\hline Leukocyte, cells/mm³ & $11.8(7.8-14.9)$ & $10.5(7.0-14.6)$ & $13.2(9.4-15.0)$ & 0.16 \\
\hline Glucose, mg/dL & 144.5 (120.6-173.8) & $142.2(117.9-175.1)$ & $147.6(128.5-169.7)$ & 0.34 \\
\hline $\mathrm{CRP}, \mathrm{mg} / \mathrm{L}$ & $9.9(2.2-59.3)$ & $9.5(2.0-52.9)$ & $14.6(2.3-68.0)$ & 0.71 \\
\hline Albumin, g/L & $33.9 \pm 4.4$ & $33.1 \pm 4.7$ & $34.9 \pm 4.0$ & 0.13 \\
\hline \multicolumn{5}{|l|}{ CSF/serum ratio } \\
\hline CSF/serum PCT ratio & $0.7(0.2-1.4)$ & $0.7(0.2-1.4)$ & $0.7(0.1-1.4)$ & 0.75 \\
\hline CSF/serum glucose ratio & $0.4(0.3-0.5)$ & $0.4(0.3-0.5)$ & $0.3(0.2-0.4)$ & 0.08 \\
\hline
\end{tabular}

The CSF and serum samples were collected on the same day. Continuous variables were expressed as mean \pm standard deviation (normally distributed), as medians and interquartile ranges (IQR, not normally distributed), which were compared using the Student $t$ test or Mann-Whitney $U$ test. The CSF and serum samples were collected on the same day

CSF cerebrospinal fluid, $P C T$ procalcitonin, CRP C-reactive protein 
with empiric antibiotic pretreatment, they were not informative any more. Our study indicated that CSF-PCT was a more robust and superior diagnostic biomarker for Gram-negative BM, even in patients who had received empiric antibiotic pretreatment.

\section{Additional file}

Additional file 1: Figure S1. Flow chart. Figure S2. Levels of CSF-PCT (a), serum PCT (b), CSF-CRP (c), serum PCT (d), CSF-leukocyte (e), and CSF-protein ( $f$ in Gram-negative and Gram-positive BM. Table S1.

Pathogens of patients with definite diagnoses of BM. Table S2. Type of

BM. Table S3. Baseline characteristics. Table S4. Predictive performance of CSF-PCT in Gram-negative BM. (DOC 452 kb)

\section{Abbreviations}

BM: Bacterial meningitis; CRP: C-reactive protein; CSF: Cerebrospinal fluid; NICU: Neurological intensive care unit; PCT: Procalcitonin

\section{Acknowledgements}

None.

\section{Funding}

Wen Jiang obtained funding from the National Natural Science Foundation of China (grant 81771406) and Wen Li obtained funding from the Science and Technology Development Project of Shaanxi Province (grant 2016SF-084) for this work.

\section{Availability of data and materials}

The datasets used and analyzed during the current study are available from the corresponding author on reasonable request.

\section{Authors' contributions}

$W L, F Y, X L S, Z H Z$, and $Y Y Z$ carried out the data collection and drafted the manuscript. WJ, WL, and FY participated in the design of the study and performed the statistical analysis. All authors read and approved the final manuscript.

\section{Ethics approval and consent to participate}

This study was based on a prospective non-interventional research on BM that registered in ClinicalTrials.gov (NCT02278016) and was approved by the ethics committee of Xijing Hospital (KY20140916-3). All procedures involving the patients complied with the ethical standards of the institutional and national research committees and with the 1975 Declaration of Helsinki and its later amendments.

\section{Consent for publication}

Yes.

\section{Competing interests}

The authors declare that they have no competing interests.

\section{Publisher's Note}

Springer Nature remains neutral with regard to jurisdictional claims in published maps and institutional affiliations.

\section{Author details}

'Department of Neurology, Xijing Hospital, Fourth Military Medical University, Xi'an 710032, People's Republic of China. ${ }^{2}$ Department of Rehabilitation Medicine, Xijing Hospital, Fourth Military Medical University, Xi'an, China.
Received: 12 December 2018 Accepted: 11 January 2019

Published online: 23 January 2019

\section{References}

1. Bassetti M, Russo A, Righi E, Dolso E, Merelli M, Cannarsa N, et al. Comparison between procalcitonin and $\mathrm{C}$-reactive protein to predict blood culture results in ICU patients. Crit Care. 2018;22:252.

2. Li W, Sun X, Yuan F, Gao Q, Ma Y, Jiang Y, et al. Diagnostic accuracy of cerebrospinal fluid procalcitonin in bacterial meningitis patients with empiric antibiotic pretreatment. J Clin Microbiol. 2017;55:1193-204.

3. Schwarz S, Bertram M, Schwab S, Andrassy K, Hacke W. Serum procalcitonin levels in bacterial and a bacterial meningitis. Crit Care Med. 2000;28:1828-32.

4. Currie J, Lin W, Zhang W. Patient knowledge and antibiotic abuse: evidence from an audit study in China. J Health Econ. 2011;30:933-49.

5. Rajs G, Finzi-Yeheskel Z, Rajs A, Mayer M. C-reactive protein concentrations in cerebral spinal fluid in Gram-positive and Gram-negative bacterial meningitis. Clin Chem. 2002;48:591-2. 